\title{
Visible Light-Enabled Paternò-Büchi Reaction via Triplet Energy Transfer for the Synthesis of Oxetanes
}

\author{
Katie A. Rykaczewski and Corinna S. Schindler* \\ Department of Chemistry, Willard Henry Dow Laboratory, University of Michigan, 930 North University Ave., Ann Arbor, \\ Michigan 48109, United States
}

Supporting Information Placeholder

\begin{abstract}
One of the most efficient ways to synthesize oxetanes is the light-enabled [2+2] cycloaddition reaction of carbonyls and alkenes, referred to as the Paternò-Büchi reaction. The reaction conditions for this transformation typically require the use of high energy UV light to excite the carbonyl, limiting the applications, safety, and scalability. We herein report the development of a visible light-mediated Paternò-Büchi reaction protocol that relies on triplet energy transfer from an iridium-based photocatalyst to the carbonyl substrates. This mode of activation is demonstrated for a variety of aryl glyoxylates and negates the need for both, visible light-absorbing carbonyl starting materials or UV light to enable access to a variety of functionalized oxetanes in up to $99 \%$ yield.
\end{abstract}

Oxetanes are four-membered oxygen-containing heterocycles that have emerged as important scaffolds in drug design and development over the past decade. The incorporation of oxetanes into active pharmaceuticals is particularly attractive and was shown to have a positive impact on their physiochemical and biochemical properties. ${ }^{1}$ Specifically, these four-membered heterocycles profoundly impact the aqueous solubility, lipophilicity, metabolic stability, and conformational preference of molecules, lending themselves as alternative scaffolds for geminal dimethyl substituents or carbonyl groups. ${ }^{2}$ Several strategies for the synthesis of functionalized oxetanes have been advanced including intramolecular etherification via nucleophilic substitution $(\mathbf{1})^{3}$, epoxide ring opening and closing $(2)^{4}$, ring contraction of carbohydrates $(3)^{5}$, and electrophilic halocyclization of alcohols (4) ${ }^{6}$ (Fig. 1A). Arguably, while each of these methods offer unique benefits to oxetane synthesis, the direct light-induced [2+2] cycloaddition between carbonyls and alkenes (5) represents a highly efficient method and the most direct approach towards these heterocycles. These $[2+2]$ cycloadditions are referred to as Paternò-Büchi reactions and allow for a variety of substitution about the heterocycle and a convergent synthesis, while requiring minimal prefunctionalization. $^{7}$

Traditionally, the Paternò-Büchi reaction proceeds via an excitation of the carbonyl moiety (6) under UV-light irradiation to subsequently engage with an alkene from the singlet or triplet excited state (7), which then engages with an alkene (8) to form oxetane 9 (Fig. 1B). Recent advances in Paternò-Büchi reactions have lent themselves to the synthesis of new and structurally complex oxetanes, but still require the use of highenergy UV-light. ${ }^{7}$ This prerequisite for UV-light is often associated with challenges in the scalability of these transformations and significant safety concerns due to possible UV radiation exposure. Despite these drawbacks, complementary visible-light induced processes are significantly less advanced although the milder reaction

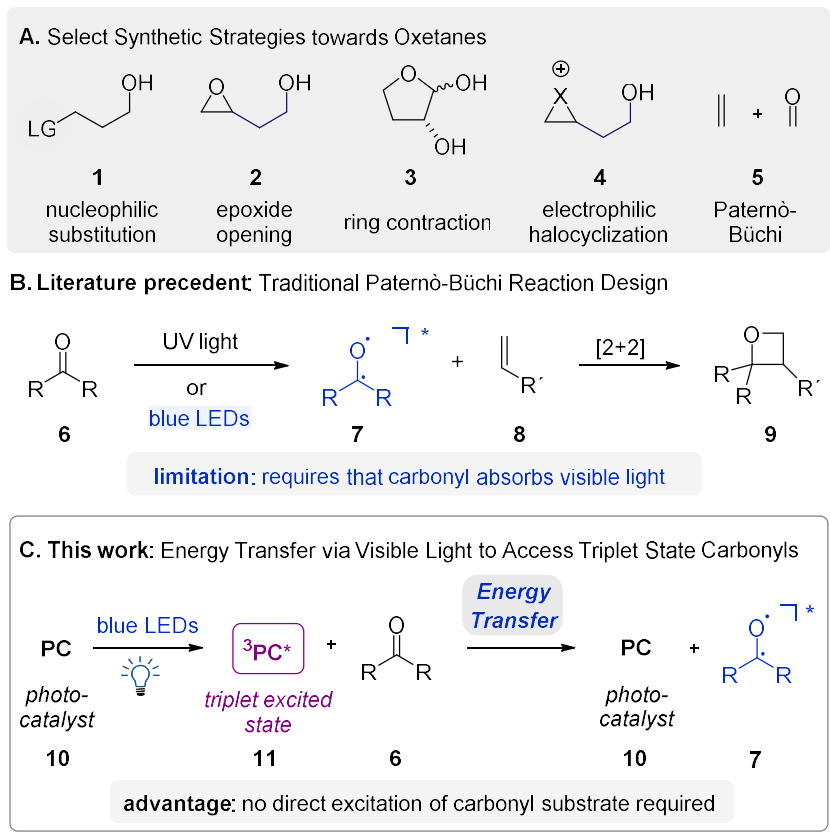

Figure 1. A. Select strategies to access oxetanes. B. Traditional Paternò-Büchi reaction design. C. Proposed energy transfer mechanism for excitation of carbonyls.

conditions are expected to significantly increase functional group tolerance, scalability, and safety of these Paternò-Büchi reactions. Importantly, two successful examples of these [2+2] cycloadditions relying on visible light irradiation have recently been reported by the groups of Dell'Amico and Ouyang. ${ }^{8-9}$

Although an important advancement, these reactions are typically dependent on substrates such as benzophenones and dicarbonyls, which absorb light in the near-UV or visible light region. Consequently, this approach is inherently limited to starting materials that incorporate either of the required photophysical features (Fig. 1B). We envisioned that a 
potentially more general synthetic approach to visible lightenabled Paternò-Büchi reactions could rely on utilizing a photocatalyst (10) to be the primary excited state species in solution to ultimately generate the desired excited state carbonyl (7) via triplet energy transfer (Fig. 1C). In related efforts, our lab has recently demonstrated that triplet energy transfer can efficiently activate functionalized imines to enable the synthesis of similarly strained four-membered nitrogencontaining heterocycles in aza Paternò-Büchi reactions. ${ }^{10}$ This transformation relies on a visible light-absorbing iridium photocatalyst to access the corresponding excited state of the substrate via triplet energy transfer, which cannot undergo direct excitation with visible light. We envisioned a similar reaction design principle to be applicable to carbonyl activation in a visible-light enabled Paternò-Büchi reaction protocol. In this reaction platform, the photocatalyst (10) is expected to absorb visible light and undergo excitation to its triplet state (11), while subsequent energy transfer to the carbonyl (6) will attain the reactive triplet energy state (7) required for efficient Paternò-Büchi reactions (Fig. 1C). Importantly, this mode of activation negates the need for visible light absorbing carbonyls, albeit requiring that their triplet energy lies close enough to that of the photocatalyst. Based on their low triplet energy (approximately $60 \mathrm{kcal} \mathrm{mol}^{-1}$ ), we hypothesized that glyoxylate derivatives could be a good starting point for the development of a visible light-enabled Paternò-Büchi reaction relying on energy transfer. ${ }^{11}$

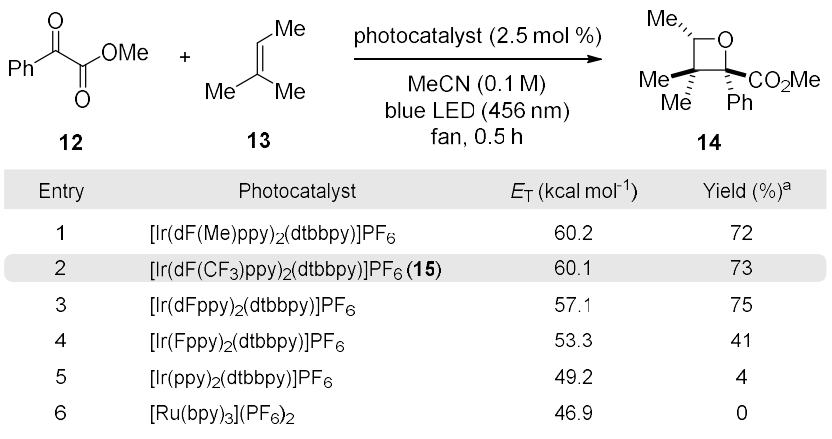

Conditions: Reactions were performed with 12 (0.05 mmol), alkene 13 (10 equiv.) and photocatalyst $(2.5 \mathrm{~mol} \%)$ in MeCN $(0.1 \mathrm{M})$ under blue LED irradiation $(456 \mathrm{~nm})$ at ambient

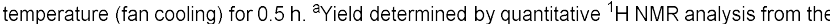
crude mixture using an internal standard

Table 1. Evaluation of photocatalysts in visible light-enabled Paternò-Büchi reactions.

Our initial investigations into the development of a mild protocol for visible light-enabled Paternò-Büchi reactions was focused on methyl benzoylformate (12), due to its superior stability compared to other glyoxylate substrates. Specifically, Norrish type II reactivity is a known competing reaction path for glyoxylate substrates under irradiation with UV-light (see Supporting Information for details). ${ }^{12}$ When glyoxylate 12 and alkene $\mathbf{1 3}$ were converted with catalytic amounts of $\left[\operatorname{Ir}(\mathrm{dF}(\mathrm{Me}) \mathrm{ppy})_{2}(\mathrm{dtbbpy})\right] \mathrm{PF}_{6}$ upon irradiation with $456 \mathrm{~nm}$ light in acetonitrile for 30 minutes, the formation of oxetane 14 was observed in $72 \%$ yield (entry 1 , Table 1 ). A selection of photocatalysts varying in their triplet energies was subsequently evaluated (entries 2-6, Table 1). Ultimately, photocatalyst 15 was identified as optimal due to its commercial availability and high yield of oxetane 14 observed (entry 2, Table 1), while photocatalysts of similar triplet energies compared to $\mathbf{1 5}$ were found equally sufficient in catalyzing the transformation.
Importantly, catalysts of lower triplet energy $\left(E_{\mathrm{T}}\right)$ failed to provide the desired oxetane product $\mathbf{1 4}$, which is consistent with a reaction mechanism relying on an energy transfer mechanism (entries 4-6, Table 1). Subsequent efforts focused on additional reaction optimization, including varying the solvent, catalyst loading, and alkene equivalents (see Supporting Information for additional details). The reaction was determined to proceed in good yields of up to $75 \%$ relying on 1,2-dichloroethane, methylene chloride, acetone, toluene, ethyl acetate, methanol, and acetonitrile as reaction solvent. Catalyst loadings as low as $0.5 \mathrm{~mol} \%$ of $\mathbf{1 5}$ and equimolar amounts of alkene $\mathbf{1 3}$ proved to be sufficient in forming oxetane 14 in up to $70 \%$ yield.

Next, we set out to investigate the scope of this visible lightenabled Paternò-Büchi reaction (Table 2). Specifically, a variety of aryl glyoxylates and different alkene reaction partners were investigated upon their ability to undergo the desired transformation. Methyl, ethyl, adamantyl, and tert-butyl esters all provided good to excellent yields of oxetanes 16-19

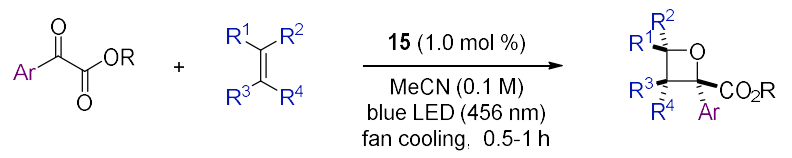

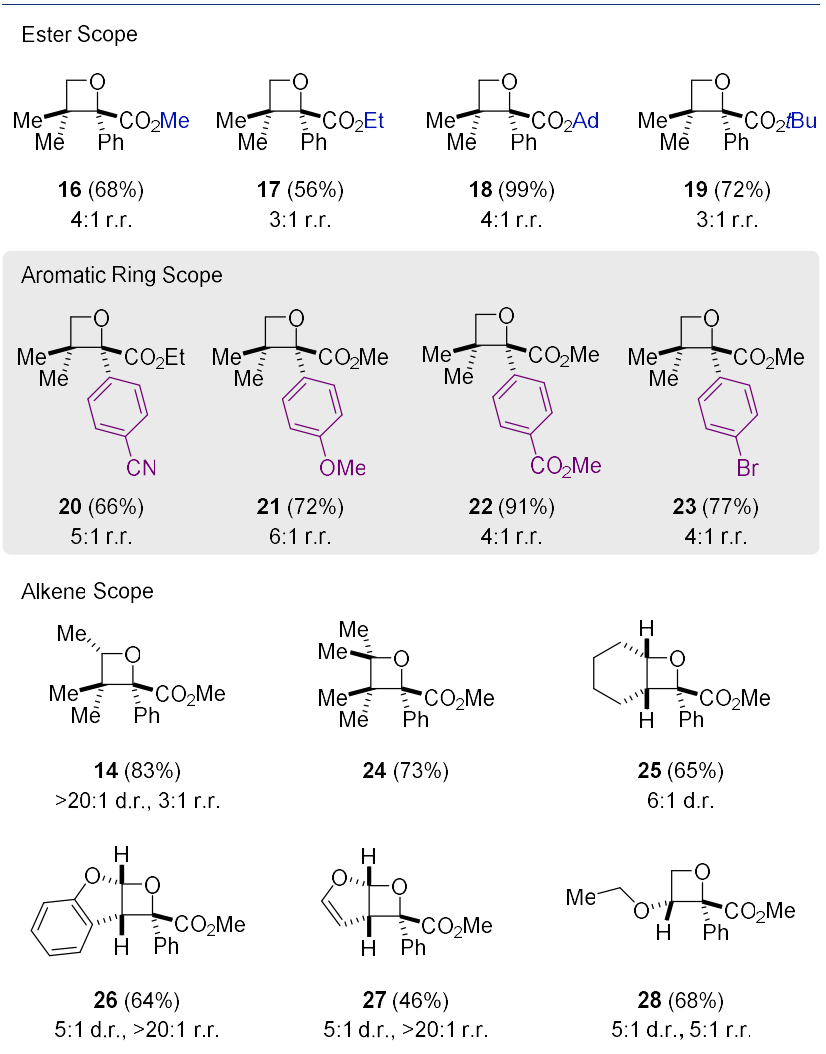

Conditions: Reactions were performed with glyoxylate substrate $(0.1 \mathrm{mmol})$, alkene $(1.5$ equiv.), and $15(1.0 \mathrm{~mol} \%)$ in $\mathrm{MeCN}(0.1 \mathrm{M})$ under blue LED irradiation $(456 \mathrm{~nm})$ at ambient temperature (fan cooling) for $0.5-1 \mathrm{~h}$; diastereomer ratios (d.r.) and regioisomer ratios (r.r.) were determined by ${ }^{1} \mathrm{H}$ NMR analysis of the crude reaction mixture; isolated yields provided

Table 2. Evaluation of the substrate scope of visible light-enabled Paternò-Büchi reactions.

of up to $99 \%$. Additionally, both electron-donating groups and electron-withdrawing groups on the aromatic ring result in the formation of the desired oxetane products. The anisole derivative 21 was obtained in $72 \%$ yield, while the electronwithdrawing para-nitrile provided oxetane 20 in $66 \%$ yield. Interestingly, an additional ester present on the aromatic ring was tolerated under the optimal reaction conditions as shown in 
the formation of oxetane $\mathbf{2 2}$ in $91 \%$ yield. Furthermore, the para-bromo aromatic glyoxylate was evaluated and proceeded in $77 \%$ yield to result in oxetane 23 . Further efforts centered on the investigation of alkene reaction partners and showed that di-, tri-, and tetrasubstituted aliphatic alkenes undergo the desired transformation providing oxetanes 14 and 24-28 in yields ranging from $46-83 \%$. Specifically, cyclohexene as an alkene partner successfully formed the bicyclic oxetane 25 in good yield of $65 \%$. Moreover, aromatic alkenes like benzofuran and furan gave the cycloadducts 26 and 27 in $64 \%$ and $46 \%$ yield, respectively. Additionally, an acyclic vinyl ether was able to provide oxetane $\mathbf{2 8}$ in $68 \%$ yield.

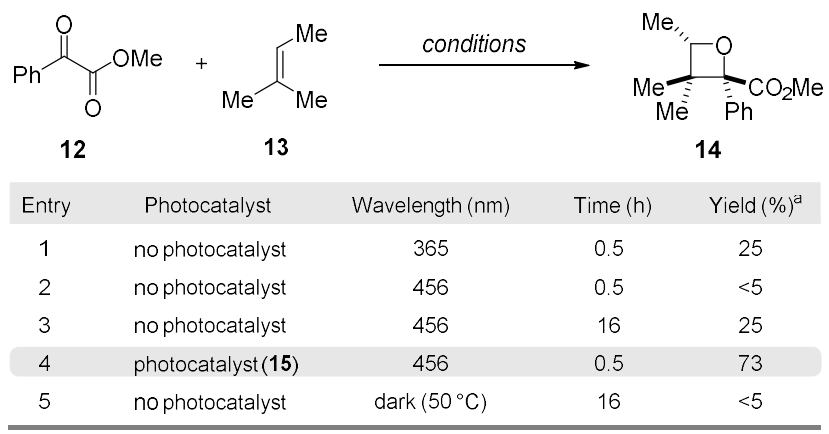

Conditions: Reactions were performed with 12 ( $0.05 \mathrm{mmol})$, alkene 13 (10 equiv.) and if indicatec photocatalyst $15(0.25 \mathrm{~mol} \%)$ in MeCN (0.1 M) under blue LED (465 $\mathrm{nm}$ ) or UV-A ( $365 \mathrm{~nm}$ ) irradiation at ambient temperature (fan cooling) for $0.5-16 \mathrm{~h}$ aYield determined by quantitative ${ }^{1} \mathrm{H}$ NMR analysis from the crude mixture using an internal standard

Table 3. Mechanistic control experiments of visible light-enabled Paternò-Büchi reactions.

Mechanistic investigations were subsequently initiated to gain additional insights into the controlling features of this visible light-enabled Paternò-Büchi reaction. Specifically, we were interested in investigating the difference between a possible direct excitation of the carbonyl and our proposed triplet energy transfer process. When glyoxylate 12 and alkene 13 were irradiated with UV-A light, the formation of oxetane 14 was observed in $25 \%$ yield after 0.5 hours (entry 1 , Table 3 ). We also tried directly irradiating the glyoxylate substrate $\mathbf{1 2}$ with visible light, however at $456 \mathrm{~nm}$, no product formation was observed within 3 hours of irradiation. After $16 \mathrm{~h}$ at $456 \mathrm{~nm}$, $25 \%$ yield of oxetane 14 was observed (entry 2, Table 3 ). Upon heating 12 and 13, without photocatalyst and light no reactivity was observed (entry 5 , Table 3 ). These results suggest that the reactivity observed under our optimal reaction conditions (entry 4 , Table 3 ), is not stemming from direct excitation of the carbonyl, and instead indicates that the photocatalyst has a significant impact on the reactivity by greatly accelerating the desired transformation. Demonstration of this reactivity suggests that other carbonyls which do not absorb visible light will be able to be sensitized with visible light in combination with a suitable photocatalyst, although a low triplet energy is required. Furthermore, a Stern-Volmer quenching study unambiguously showed that glyoxylate $\mathbf{1 2}$ productively quenches the photocatalyst (15) (see Supporting Information for details). We postulate that a photoredox process is unlikely under the optimized reaction conditions as the excited state redox potentials of $\mathbf{1 5}\left(\mathrm{Ir}^{\mathrm{III} / \mathrm{II}}=+1.21 \mathrm{~V}\right.$ versus $\mathrm{SCE}$; $\mathrm{Ir}^{\mathrm{IV} / \mathrm{III}}{ }^{*}=-$ $0.89 \mathrm{~V}$ versus SCE) are not expected to be sufficient for an effective oxidation or reduction of glyoxylate $12\left(E_{\mathrm{red}}=-1.16\right.$ $\mathrm{V}$ versus SCE; $E_{\text {red }}=-1.75 \mathrm{~V}$ versus SCE, see Supporting Information for additional details). ${ }^{13}$ Based on these combined results, we propose a triplet energy transfer mechanism to be operative under our optimal reaction conditions for PaternòBüchi reactions in which the carbonyl triplet state is accessible via energy transfer from an excited state photocatalyst (Figure 2).

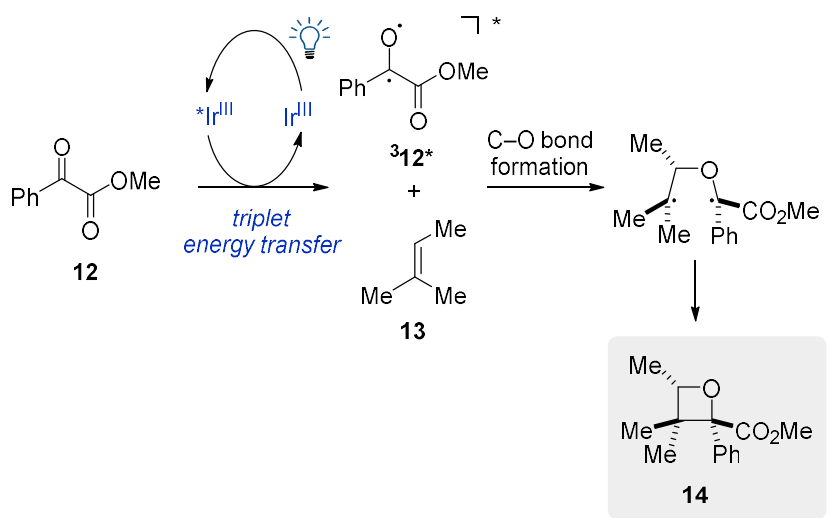

Figure 2. Proposed triplet energy transfer mechanism for the synthesis of oxetane 14.

We herein report the development of a visible light-mediated Paternò-Büchi reaction between carbonyls and alkenes relying on triplet energy transfer. Importantly, the reaction is greatly accelerated in the presence of a suitable photocatalyst and demonstrates the capability of visible-light absorbing photocatalysts to efficiently transfer energy to carbonyls. This reaction platform further expands the toolbox for medicinal chemists interested in modulating drug properties through the incorporation of functionalized oxetanes, as this method negates the need for UV light and proceeds rapidly under the optimized reaction conditions.

\section{ASSOCIATED CONTENT}

\section{Supporting Information}

The Supporting Information is available free of charge on the ACS Publications website.

Experimental details and spectroscopic data for all new reactants and products (PDF)

\section{AUTHOR INFORMATION}

\section{Corresponding Author}

*E-mail: corinnas@umich.edu

ORCID

Katie A. Rykaczewski: 0000-0001-6914-7180

Corinna S. Schindler: 0000-0003-4968-8013

\section{Author Contributions}

The manuscript was written through contributions of all authors. All authors have given approval to the final version of the manuscript.

\section{ACKNOWLEDGMENTS}

We thank the University of Michigan Office of Research and the NIH/National Institute of General Medical Sciences (R01GM118644) for financial support. C.S.S. thanks the David and Lucile Packard Foundation, the Alfred P. Sloan Foundation, and the Camille and Henry Dreyfus Foundation. K.A.R. thanks the National Science Foundation for a Graduate Research Fellowship.

\section{REFERENCES}


(1) Bull, J. A.; Davis, O. A.; Doran, R.; Morgan, K. F. Oxetanes: Recent Advances in Synthesis, Reactivity, and Medicinal Chemistry. Chem. Rev. 2016, 116, 12150-12233.

(2) (a) Wuitschik, G.; Carreira, E. M.; Wagner, B.; Fischer, H.; Parrilla, I.; Schuler, F.; Rogers-Evans, M.; Müller, K. Oxetanes in Drug Discovery: Structural and Synthetic Insights. J. Med. Chem. 2010, 53, 3227-3246. (b) Burkhard, J. A.; Wuitschik, G.; Rogers-Evans, M.; Müller, K.; Carreira, E. M. Oxetanes as Versatile Elements in Drug Discovery and Synthesis. Angew. Chem. Int. Ed. 2010, 49, 9052-9067.

(3) For examples on intramolecular etherification: (a) Picard, P.; Leclercq, D.; Bats, J.-P.; Moulines, J. An Efficient One-Pot Synthesis of Oxetanes from 1,3-Diols. Synthesis 1981, 1981, 550-551. (b) Servrin, M.; Krief, A. Regioselective and [C,C] Connective Routes to Oxetane and Tetrahydrofuranes. Tetrahedron Lett. 1980, 21, 585-586.

(4) For examples on epoxide opening and closing: Okuma, K.; Tanaka, Y.; Kaji, S.; Ohta, H. Reaction of Dimethyloxosulfonium Methylide with Epoxides. Preparation of Oxetanes. J. Org. Chem. 1983, 48, 5133-5134.

(5) For examples on carbohydrate contraction: (a) Jenkinson, S. F.; Fleet, G. W. J. Oxetanes from the Ring Contraction of $\alpha$ Triflates from $\gamma$-Lactones: Oxetane Nucleosides and Oxetane Amino Acids. Chimia 2011, 65, 71-75. (b) Austin, G. N.; Fleet, G. W. J.; Peach, J. M.; Prout, K.; Son, J. C. Chiral Oxetanes from Sugar Lactones: Synthesis of Derivatives of 3,5-Anhydro-1,2-O-Isopropylidine- $\alpha$-D-Glucuronic Acid and of 3,5-Anhydro-1,2-O-Isopropylidine- $\beta$-L-Iduronic Acid. Tetrahedron Lett. 1987, 28, 4741-4744.

(6) For examples on halocyclization: (a) Ehlinger, E.; Magnus, P. Silicon in Synthesis. 10. The (Trimethylsilyl)allyl Anion: A $\beta$ Acyl Anion Equivalent for the Conversion of Aldehydes and Ketones into $\gamma$-Lactones. J. Am. Chem. Soc. 1980, 102, 5004-5011. (b) Manabe, S.; Nishino, C. Stereochemistry of cis-Clerodane Diterpenes. Tetrahedron 1986, 42, 3461-3470.

(7) Paternò-Büchi: (a) Paternò, E.; Chieffi: G. Synthesis in Organic Chemistry Using Light. Note II. Compounds of Unsaturated Hydrocarbons with Aldehydes and Ketones. Gazz. Chim. Ital. 1909, 39, 341. (b) Büchi, G.; Inman C. G.; Lipinsky E. S. Light-Catalyzed Organic Reactions. I. The Reaction of Carbonyl Compounds with 2-methyl-2-butene in the Presence of Ultraviolet Light. J. Am. Chem. Soc. 1954, 76, 4327. (c) Bach, T. Stereoselective Intermolecular [2+2]Photocycloaddition Reactions and Their Application in Synthesis. Synthesis 1998, 5, 683-703. (d) Abe, M. Recent Progress Regarding Regio-, Site-, and Stereoselective Formation of Oxetanes in Paterno-Buchi Reactions. J. Chin. Chem. Soc. 2008, 55, 479-486. (e) D'Auria, M.; Racioppi, R. Oxetane Synthesis Through the Paternò-Büchi Reaction. Molecules 2013, 18, 11384-11428.

(8) Mateos, J.; Vega-Peñaloza, A.; Franceschi, P.; Rigodanza, F.; Andreetta, P.; Companyó, X.; Pelosi, G.; Bonchio, M.;
Dell'Amico, L. A Visible-Light Paternò-Büchi Dearomatisation Process Towards the Construction of OxetoIndolic Polycycles. Chem. Sci. 2020, 11, 6532-6538.

(9) Li, H. F.; Cao, W.; Ma, X.; Xie, X.; Xia, Y.; Ouyang, Z. Visible-Light-Driven [2 +2$]$ Photocycloadditions between Benzophenone and $\mathrm{C}=\mathrm{C}$ Bonds in Unsaturated Lipids. J. Am. Chem. Soc. 2020, 142, 3499-3505.

(10) (a) Becker, M. R.; Richardson, A. D.; Schindler, C. S. Functionalized Azetidines Via Visible Light-Enabled Aza Paternò-Büchi Reactions Nat. Commun. 2019, DOI: 10.1038/s41467-019-13072-x. (b) Becker, M. R.; Wearing, E. R.; Schindler, C. S. Synthesis of Azetidines via Visible LightMediated Intermolecular [2+2] Photocycloaddition. ChemRxiv 2020, DOI: 10.26434/chemrxiv.11832993.v1

(11) Herkstroeter, W. G.; Lamola, A. A.; Hammond, G. S. Mechanisms of Photochemical Reactions in Solution. XXVIII. Values of Triplet Excitation Energies of Selected Sensitizers. J. Am. Chem. Soc. 1964, 86, 4537-4540.

(12) (a) Hu, S.; Neckers, D. C. Rapid Regio- and Diastereoselective Paternò-Büchi Reaction of Alkyl Phenylglyoxylates. J. Org. Chem. 1997, 62, 564-567. (b) Hu, S.; Neckers, D. C. Photochemical Reactions of Alkenyl Phenylglyoxylates. $J$. Org. Chem. 1997, 62, 6820-6826. (c) Hu, S.; Neckers, D. C. Photochemical Reactions of Alkyl Phenylglyoxylates. J. Org. Chem. 1996, 61, 6407-6415.

(13) Teegardin, K.; Day, J. I.; Chan, J.; Weaver, J. Advances in Photocatalysis: A Microreview of Visible Light Mediated Ruthenium and Iridium Catalyzed Organic Transformations. Org. Process Res. Dev. 2016, 20, 1156-1163. 


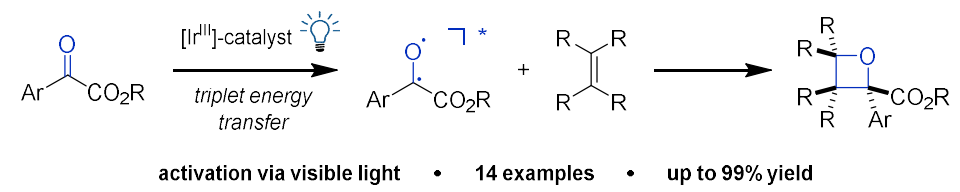

